\title{
Implications of a Rotating Mass Matrix
}

\author{
José BORDES \\ jose.m.bordes@ uv.es \\ Departament Fisica Teorica, Universitat de Valencia, \\ calle Dr. Moliner 50, E-46100 Burjassot (Valencia), Spain \\ CHAN Hong-Mo \\ chanhm@ @2.rl.ac.uk \\ Rutherford Appleton Laboratory, \\ Chilton, Didcot, Oxon, OX11 0QX, United Kingdom \\ TSOU Sheung Tsun \\ tsou@ maths.ox.ac.uk \\ Mathematical Institute, University of Oxford, \\ 24-29 St. Giles', Oxford, OX1 3LB, United Kingdom
}

\begin{abstract}
The fermion mass matrix, in addition to having eigenvalues (masses) which run, also changes its orientation (rotates) with changing energy scales. This means that its eigenstates at one scale will no longer be eigenstates at another scale, leading to effects where fermions of different flavours can "transmute" into one another. In this paper, the implications of a rotating mass matrix are analysed and possible transmuation effects are investigated both in the Standard Model (SM) and in the so-called Dualized Standard Model (DSM) that we advocate, arriving at the conclusion that some transmutational decays such as $\psi \longrightarrow \mu \tau, \Upsilon \longrightarrow \mu \tau$ or $\pi^{0} \longrightarrow e \mu$ may be within experimental range, if not immediately, then in the near future.
\end{abstract}




\section{Introduction - the Rotating Mass Matrix}

By a rotating mass matrix, we mean one which undergoes unitary transformations through scale changes.

As most quantities in quantum field theory vary under changing scales, in particular the masses of particles or in other words the eigenvalues of mass matrices, it is no surprise that the orientations of mass matrices may also change.

Indeed, even in the Standard Model as usually conceived, nontrivial mixing between up and down fermion states will generally induce rotating mass matrices. That this is so can be seen from the following renormalization group equations:

$$
\begin{aligned}
16 \pi^{2} \frac{d U}{d t} & =\frac{3}{2}\left(U U^{\dagger}-D D^{\dagger}\right) U+\left(\Sigma_{u}-A_{u}\right) U, \\
16 \pi^{2} \frac{d D}{d t} & =\frac{3}{2}\left(D D^{\dagger}-U U^{\dagger}\right) D+\left(\Sigma_{d}-A_{d}\right) D .
\end{aligned}
$$

satisfied by the mass matrices $U$ and $D$ of respectively the up and down fermions [1]. Nontrivial mixing between up- and down-states means that the matrices $U$ and $D$ are related by a nondiagonal matrix $D=V U V^{\dagger}$ or $U=V^{\dagger} D V$, so that $U$ and $D$ cannot be simultaneously digonalized. Suppose now at some $t$ we diagonalize $U$ in (1.1) by an appropriate unitary transformation. All other terms in (1.1) are then diagonal but not the term $D D^{\dagger} U$. Hence, this term will necessarily de-diagonalize the matrix $U$ on running by (1.1) to a different $t$, or in other words the matrix $U$ will rotate with changing $t$ [2]. Similar arguments hold also for the rotation of the matrix $D$. Given that quarks have long been known to have a nontrivial (CKM) mixing matrix [3], and that recent experiments on neutrino oscillation [4, 5, 6] strongly suggest a nontrivial (MNS) mixing [7] also for leptons, we have to conclude that the mass matrices for both quarks and leptons will rotate with changing scales even if there are no forces and interactions in nature other than those currently studied in the Standard Model.

However, there is clearly a possibility-perhaps one might even say a strong theoretical reason to suppose - that forces may exist in nature other than those now conventionally studied in the Standard Model, which can give further rotations to the fermion mass matrices. Indeed, in the usual formulation of the Standard Model, the fact that there are 3 generations of fermions and that they mix is taken as an input from experiment, with the result that the framework depends on a large number of empirical parameters, about 
three-quarters of which are traceable to the mystery of generations. One could thus hope that in future when the generation puzzle is solved, some or even most of the empirical parameters appearing in the present Standard Model will be predictable. One favourite and perhaps most natural assumption for theoretical attempts in this direction is that generations originate as a broken 'horizontal' gauge symmetry [8]. If that is the case, then the interactions associated with this symmetry which mix the generation index are likely also to rotate the fermion mass matrices in generation space. This rotation would be over and above that driven by the nondiagonal CKM or MNS matrices via the mechanism described in the preceding paragraph.

Whatever its origin, however, the rotation of fermion mass matrices is theoretically of the same standing as the running of masses and coupling constants, and if testable by experiment would lend equal support to the quantum field theory framework. Moreover, if experiment shows that the rotation is different from simply that given by renormalization group equations (1.1) and (1.2) via the empirical mixing matrix, then it suggests that there are forces at work other than those currently studied in the Standard Model which are of the type considered in the preceding paragraph. This means that we would have opened up a new window for investigations furthering our long-standing quest for the origin of fermion generations. And since, as we shall see, mass matrix rotation leads to an entirely new and very distinctive category of physical phenomena, there is a fair chance of its effects being observed by experiment in the not too distant future. The purpose of the present paper is to explore such possibilities.

\section{Specification of Fermion States}

The fact that the mass matrix rotates poses immediately a question of physical interpetation at a rather basic level, namely the question of how to define the state vectors of the various fermion states, and hence the mixing matrices between them. For the familiar case of a non-rotating mass matrix, one defines the state vectors of the 3 generations as its eigenvectors, which are by assumption scale-independent and, since the matrix is hermitian, are also mutually orthogonal as they should be if they are to represent independent physical entities. The mixing matrix between the up and down fermion types, being the overlap matrix between 2 scale-independent orthonormal triads of state vectors, one for each type, is then also scale-independent and automatically unitary. Only the eigenvalues of a mass matrix then run, and 
the masses of the 3 states (generations) can be defined as the running values each evaluated at the scale equal to its value. However, when the mass matrix rotates, although one can still diagonalize the matrix at any scale by a triad of mutually orthogonal eigenvectors, this triad will be scale-dependent, and it is not immediately clear which vectors at which scale(s) are to be identified as the state vectors of the 3 generations. Although the ambiguity may not in all cases be numerically significant in view of present limitations in experimental accuracy, it still has to be resolved as a matter of principle against the day when better accuracy is achieved.

Now, the state vector of a physical state like the muon is normally, we think, taken as a scale-independent concept, or otherwise we are likely to meet with some awkwardness in its physical interpretation. For example, suppose we were to define the muon state as the eigenvector with the second highest eigenvalue of the charged lepton mass matrix at any scale. Then since the matrix rotates, the $\mu$ state vector will point at different scales in different directions in generation space. In that case, the muons obtained say from an energetic beam of charged $\pi$ 's, which we know decay almost entirely into $\mu$ 's at rest, will no longer appear as purely muons when it hits the target, but as a linear combination of $e, \mu$ and $\tau$, which is at variance with what is usually understood. Although one can in principle insist on defining fermion states as the eigenstates of the rotating mass matrix at every scale, and so long as one is consistent in their interpretation one would arrive in the end at the same physical results, such a procedure would seem to be rather inconvenient. In this paper therefore, we opt for a scale-independent definition of fermion state vectors, in which case it would be incumbent upon us to specify exactly the scale at which each of these vectors are to be defined.

If the rotation of the mass matrix is negligible to a certain approximation below some scale, then one may define to this approximation the state vectors as the orthonormal triad of eigenvetors of the mass matrix taken at that scale. This, though seldom stated explicitly is, we think, the tacit criterion usually adopted in the literature which we shall refer to in this paper as Fixed Scale Diagonalization FSD. It is, of course, at best an approximation for the mass matrix cannot stop rotating abruptly, and that approximation, as we shall see later, may not be all that good in certain circumstances. Besides, this criterion involves a degree of arbitrariness in choosing a certain fixed scale to effect the diagonalisation of the mass matrix, and begs the question of principle why it should be that particular scale and not some other.

In view of this, one may be tempted instead to do the following. As one defines the mass of a state as the eigenvalue evaluated at the scale equal 
to its value, one may try to define as its state vector the corresponding eigenvector also at the same scale. This would be more democratic and less arbitray than the FSD criterion but it will not work. Consider for example the $U$-type quarks. Following the above proposal, one would then define the $t$ state vector as the eigenvector with the largest eigenvalue $m_{3}$ of the matrix evaluated at the scale $\mu=m_{3}(\mu)$, the $c$ state vector as the eigenvector with the second largest eigenvalue $m_{2}$ at the scale $\mu=m_{2}(\mu)$, and the $u$ state vector as the eigenvector with the smallest eigenvalue $m_{1}$ at the scale $\mu=m_{1}(\mu)$. These 3 state vectors, however, will not be mutually orthogonal, for although the 3 eigenvectors of the hermitian mass matrix are mutually orthogonal when all evaluated at the same scale, they have no reason to be so when evaluated each at a vastly different scale since the matrix by assumption rotates with changing scales. It thus contradicts the assertion that they represent 3 independent physical entities; in particular, it would imply that the mixing (CKM or MNS) matrix, being a transformation matrix between the 2 triads of up and down state vectors, is not unitary as it ought to be.

There is, however, a working criterion for defining state vectors each at its own mass scale along the lines suggested in the above paragraph which takes account of the rotation between the different mass scales yet still gives mutually orthogonal state vectors and hence a unitary mixing matrix. This was first proposed by us [9] in connection with a scheme for quark and lepton mixing which we call the Dualized Standard Model (DSM), but the criterion can in fact be applied to define state vectors in any scheme with a rotating mass matrix [10]. For 3 generations, the criterion goes as follow. We run the mass matrix $m$ down in scale until we have for its highest eigenvalue $m_{3}$ a solution to the equation $\mu=m_{3}(\mu)$. This value at this scale we define as the mass $m_{3}$, and the corresponding eigenvector the state vector $\mathbf{v}_{\mathbf{3}}$ of the heaviest generation. Below that energy, the state 3 no longer exists as a physical state, and only the two lighter generations survive, whose state vectors have to be orthogonal to $\mathbf{v}_{\mathbf{3}}$. We define then the mass matrix at energies below $m_{3}$ as the $2 \times 2$ submatrix $\hat{m}$ of $m$ in the subspace orthogonal to $\mathbf{v}_{\mathbf{3}}$. To find now the mass and state vector for generation 2 , we follow with $\hat{m}$ the same procedure used above with $m$ for generation 3 and run $\hat{m}$ down in scale until we find a solution to the equation $\mu=\hat{m}_{2}(\mu)$, which value we call the mass $m_{2}$ and the corresponding eigenvector at that scale the state vector $\mathbf{v}_{\mathbf{2}}$ of the generation 2 . The state vector of the lightest generation 1 is now also defined as the vector orthogonal to both $\mathbf{v}_{\mathbf{3}}$ and $\mathbf{v}_{\mathbf{2}}$, while the mass of 1 is obtained by repeating the same procedure, namely by running down in 
scale the remaining $1 \times 1$ submatrix, namely the expectation value $\left\langle\mathbf{v}_{\mathbf{1}}|m| \mathbf{v}_{\mathbf{1}}\right\rangle$, until its value equals the scale. In this way, each mass is evaluated at its own appropriate scale while the physical state vectors of the 3 generations are mutually orthogonal as they should be. Furthermore, the mixing matrix, taken as the overlap matrix between the triads of state vectors so defined for the up and down states, will be both unitary and scale-independent. This criterion for defining state vectors, which is the only one we are aware of with the required properties, we shall refer to in future as Step-by-Step Diagonalization SSD.

\section{Fermion Transmutation as Consequence}

Whether one accepts the SSD proposal for defining physical fermion state vectors or chooses to ignore these subtleties and adopts the approximate criterion of FSD, the fact remains that the mass matrix will continue to rotate at high energy, and so long as the state vectors are defined as the eigenvectors diagonalizing the mass matrix at some prescribed scale(s), they will generally no longer diagonalize the mass matrix at some higher energy scales. Now, the fermion mass matrix appears for example in the fermion propagator of Feynman diagrams, and if it is nondiagonal, so also will be the transition amplitudes represented by these diagrams. Physically, this means that transitions will be induced in which a particle of one generation converts into a particle of another generation. For example, the diagram of Figure 1 ,

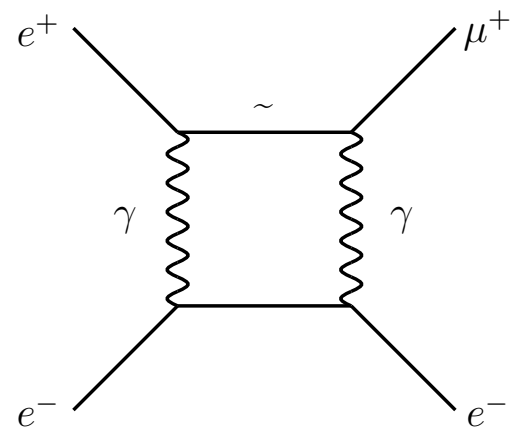

Figure 1: Transmutation of $e$ into $\mu$ in $e^{+} e^{-}$collision. 
where a tilde denotes the occurrence of a nondiagonal fermion mass matrix, will have an off-diagonal element linking the $e$ state vector to the $\mu$ state vector, leading to a $e^{+} e^{-} \longrightarrow \mu^{+} e^{-}$transition.

Such a conversion of $e$ to $\mu$ is quite distinctive and singular, differing from that induced, for example, by a flavour-changing neutral current (FCNC) exchanged between the initial $e^{+}$and $e^{-}$. The latter will normally produce in addition to the $\mu^{+}$at the upper vertex also a $\mu^{-}$at the lower vertex, giving rise to a "double conversion", although under certain circumstances where the exchanged FCNC bosons themselves mix, "single conversion" can also occur, as for example in the situation considered in [13]. However, even in that last case, the conversion rate depends on the masses and couplings of the exchanged FCNC bosons which are still rather elusive quantitites, whereas the conversion effect considered here is due only to the rotation of the mass matrix, and may therefore be quite restricted. We propose therefore to call the latter "transmutation" to distinguish it from other conversion phenomena.

The effect of transmutation is so unique as a consequence of the rotating mass matrix that if it occurs with appreciable cross sections, it should be identifiable in experiment with little difficulty. At first sight, therefore, the prediction looks alarming, for the conservation of such quantities as muon number have been checked already to high accuracy, and their wholesale violation as suggested above seems hardly likely to survive the existing experimental bounds. On closer examination, however, the conclusion becomes less obvious, mainly for the following reason. At low energy, near the scale(s) at which the mass matrix is diagonalized to define the physical fermion states, as explained in the preceding section, the deviations of the mass matrix from diagonality are small, and so also will be the cross sections for transmutation. At high energy, on the other hand, where the off-diagonal mass matrix elements can become sizeable, it turns out that transmutation cross sections will be suppressed, as can be seen in the following example.

One of the simplest transmutation processes that one can think of is what one can call the photo-transmutation of leptons, which to leading order is given just by the Feynman diagrams in Figure 2 which are the same as for ordinary Compton scattering except that the fermion line here carries a rotating and generally non-diagonal mass matrix. Consider first the $s$ channel diagram (a) in which the lepton propagator $1 /(\not p+\not k-m)$ appears, carrying the non-diagonal mass matrix $m$ where transmutation originates. For $s=(p+k)^{2}$ large, we can expand the propagator in powers of $m / \sqrt{s}$, 


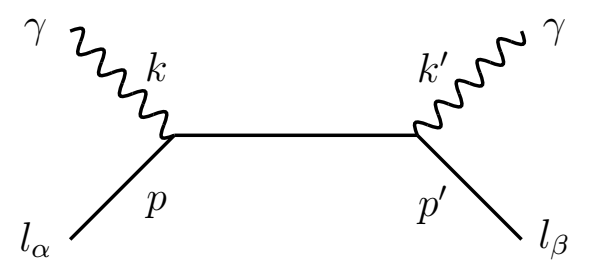

(a)

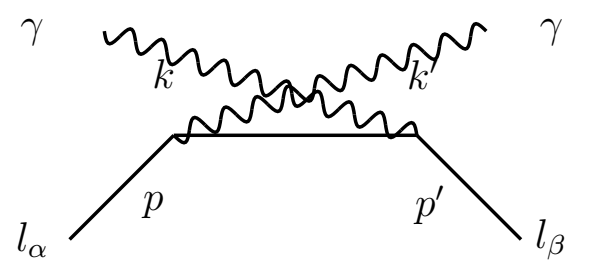

(b)

Figure 2: Feynman diagrams for photo-transmutation of leptons.

obtaining symbolically:

$$
\frac{1}{(\not p+\not k-m)} \sim \frac{1}{(\not p+\not k)}\left(1+\frac{m}{\sqrt{s}}\right)
$$

where one sees that the off-diagonal terms leading to transmutation is suppressed by a factor of $1 / \sqrt{s}$. Similarly, the lepton propagator in the diagram (b) for $u$ large can be approximated as:

$$
\frac{1}{\left(\not p-\not k^{\prime}-m\right)} \sim \frac{1}{\left(\not p-\not k^{\prime}\right)}\left(1+\frac{m}{\sqrt{|u|}}\right)
$$

leading to transmutation effects suppressed by a factor $1 / \sqrt{|u|}$. In the region where $|u|$ is small, off-diagonal contributions can remain sizeable, but the size of this region itself decreases as $1 / s$ so that the integrated effect remains small.

For these reasons, transmutation effects arising from the rotating mass matrix are seldom very large and the question whether they can already be ruled out by existing data, and if not, how soon, and under what conditions, they will be verifiable, is not immediately obvious and can only be answered by detailed analysis. The answers will depend on the process investigated and on the speed with which the mass matrices rotate, and these in turn depend on the theory or model one is considering. We examine below and in a companion paper [14 some specific examples.

\section{Two Rotation Mechanisms}

In this section, we investigate the rotating fermion mass matrix in 2 specific schemes at, as it were, opposite ends of the spectrum so as hopefully to 
span most reasonable possibilities. At one end of the spectrum, we assume that there are no forces in nature other than those currently studied in the Standard Model so that all the rotation in the fermion mass matrix is driven by the electroweak Higgs terms via the empirical nondiagonal mixing (CKM or MNS) matrix. The generation phenomenon is thereby left unexplained. Fermion state vectors are to be specified by the FSD prescription (to contrast with the scheme below which only works with the SSD prescription) in which the mass matrix is diagonalized at some chosen (low) scale. In the case of leptons, since some mass and mixing parameters are but poorly known, these specifications have yet to be supplemented by further assumptions based on current popular views in a manner to be detailed later. We shall refer to this henceforth as the NSM scheme (for naively implemented Standard Model). At the other end of the spectrum, we consider a scheme we ourselves suggested [10], which in turn was abstracted from our so-called Dualized Standard Model (DSM) 9, 12 although it need not strictly adhere to the duality concepts of that earlier work. In this scheme, generation itself originates from a broken $S U(3)$ gauge symmetry, the rotation is driven by Higgs bosons associated with the breaking of this symmetry, and even the nondiagonal mixing matrices as experimentally observed are themselves consequences of the mass matrix rotation. Here the SSD prescription for defining state vectors is essential. Given that in the DSM it is the rotation which gives rise to fermion mixing, and not the other way round as in the NSM case with the mixing driving the rotation, transmutation effects are usually, though not in all cases, larger in the DSM than in the NSM, and hence would be easier either to disprove or to confirm.

Our first task is to evaluate the rotating mass matrix over a range of scales, say, up to the energy achievable in the foreseeable future of around 100 $\mathrm{TeV}$. For the NSM case, this in principle requires the solution of the equations (1.1) and (1.2) together with any other renormalization group equations they are coupled to, which though standard is a little involved. For our present purpose of a first exploration, however, we may approximate by linearizing the equations and keeping only those terms actually driving the rotation, namely the term $D D^{\dagger} U$ in (1.1) and the term $U U^{\dagger} D$ in (1.2). We need as input from experiment the masses of the fermion states and the mixing matrices measured at low energies. Assuming that the scale in the FSD prescription chosen for defining the fermion state vectors coincides with the scale at which the empirical mixing matrices are measured, the rotating mass matrices can then be evaluated by iterating the two equations. For quarks, both the masses and the CKM matrix have now been determined to sufficient 
accuracy for our purpose and pose no practical difficulty. This calculation has been performed but since it will not be of use in what follows in this paper, the result will not be presented. For leptons, however, the MNS matrix is still poorly known, while the Dirac masses of neutrinos on which their Higgs couplings depend are still almost entirely unconstrained. For these, therefore, we shall just insert for our exploration the most popular theoretical biases at present, namely for the MNS matrix the so-called bi-maximal mixing [15] version:

$$
U_{M N S}=\left(\begin{array}{ccc}
1 / \sqrt{2} & 1 / \sqrt{2} & 0 \\
1 / 2 & 1 / 2 & 1 / \sqrt{2} \\
1 / 2 & 1 / 2 & 1 / \sqrt{2}
\end{array}\right),
$$

and for the Dirac mass $m_{3}$ of the heaviest neutrino a value around the mass of the $t$ quark. One shall need the Dirac mass $m_{2}$ of the second heaviest neutrino also, which is taken, for lack of any better choice, to be of the order of the charm mass. Since the equations have been linearized in approximation, the result of the rotation for charged leptons can be summarized simply by giving the rates of change of the off-diagonal elements of the ('hermitized') mass matrix with the logarithm of the energy, which are explicitly given as:

$$
\frac{d L}{d t}=\frac{3}{128 \pi^{2}} \frac{1}{V^{2}}\left(\begin{array}{ccc}
2 m_{2}^{2} m_{e} & \frac{1}{\sqrt{2}} m_{2}^{2}\left(m_{e}+m_{\mu}\right) & \frac{1}{\sqrt{2}} m_{2}^{2}\left(m_{e}+m_{\tau}\right) \\
\frac{1}{\sqrt{2}} m_{2}^{2}\left(m_{e}+m_{\mu}\right) & 2 m_{3}^{2} m_{\mu} & m_{3}^{2}\left(m_{\mu}+m_{\tau}\right) \\
\frac{1}{\sqrt{2}} m_{2}^{2}\left(m_{e}+m_{\tau}\right) & m_{3}^{2}\left(m_{\mu}+m_{\tau}\right) & 2 m_{3}^{2} m_{\tau}
\end{array}\right)
$$

with $V$ being the vev of the electroweak Higgs, i.e. $246 \mathrm{GeV}$. Putting in then the suggested values of the masses, one obtains that $\langle\mu|m| \tau\rangle$ changes by about $5.5 \times 10^{-3},\langle e|m| \tau\rangle$ by about $1.77 \times 10^{-7}$ and $\langle e|m| \mu\rangle$ by about $1.06 \times 10^{-8} \mathrm{GeV}$ per decade change in energy. These results will be useful later for estimating the rates of various transmutation effects implied by the NSM scheme. One notices that in (4.2), $\langle e|m| \tau\rangle$ is proportional to $m_{2}^{2}$ only by virtue of the assumed zero in the bi-maximal mixing matrix. If that element $U_{e 3}$ of the MNS matrix is nonzero, then $\langle e|m| \tau\rangle$ would be proportional to $m_{3}^{2}$, which in turn can lead on iteration to terms of order $m_{3}^{4}$ in the element $\langle e|m| \mu\rangle$, thus making both much larger than the above estimates, which represent therefore a sort of lower limit.

For the DSM case, the calculation, though more intricate, has been more explicitly specified. As the scheme depends on only 3 parameters [10 which have already been determined by fitting to some and then used to give very sensible predictions to others of the mass and mixing parameters of both quarks and leptons, no further information needs to be supplied. Indeed, 
in making these fits and predictions, the rotating mass matrices for quarks and leptons had perforce already been evaluated, though not previously presented. The result for the charged leptons which is of concern to us in this paper is now given in Figures 3 .

To check our result, without going through the details of the fit in [10], one needs only to iterate the following equation for a vector $\left(x^{\prime}, y^{\prime}, z^{\prime}\right)$ :

$$
\frac{d}{d t}\left(\begin{array}{l}
x^{\prime} \\
y^{\prime} \\
z^{\prime}
\end{array}\right)=\frac{5}{32 \pi^{2}} \rho^{2}\left(\begin{array}{c}
x_{1}^{\prime} \\
y_{1}^{\prime} \\
z_{1}^{\prime}
\end{array}\right)
$$

with

$$
x_{1}^{\prime}=\frac{x^{\prime}\left(x^{\prime 2}-y^{\prime 2}\right)}{x^{\prime 2}+y^{\prime 2}}+\frac{x^{\prime}\left(x^{\prime 2}-z^{\prime 2}\right)}{x^{\prime 2}+z^{\prime 2}}, \quad \text { cyclic, }
$$

starting from some initial value $\left(x_{I}, y_{I}, z_{I}\right)$ with a coupling $\rho$, all given in eq. 16 of [10]. For an accuracy of about a percent, some 500 iterations are needed per decade change in energy, where the vector $\left(x^{\prime}, y^{\prime}, z^{\prime}\right)$ is to be normalized after every iteration. The resulting mass matrix is of the factorized form:

$$
m^{\prime}=m_{T}\left(\begin{array}{c}
x^{\prime} \\
y^{\prime} \\
z^{\prime}
\end{array}\right)\left(x^{\prime}, y^{\prime}, z^{\prime}\right)
$$

where $m_{T}$ is a normalization depending on the fermion-type $T$, which may be taken as the mass of the heaviest generation state of that type. In (4.5), the mass matrix is given with respect to a fixed (gauge) basis in generation space, but the matrix with respect to the physical basis as presented in, for example, Figure 3 is also easily evaluated with the (physical) state vectors determined in accordance with the SSD prescription outlined in section 2 . Thus, the physical state vectors of the charged leptons in the gauge basis are, explicitly:

$$
\begin{aligned}
& \mathbf{v}_{\tau}=(0.9967,0.0760,0.0268) \\
& \mathbf{v}_{\mu}=(-0.0759,0.7741,0.6285) \\
& \mathbf{v}_{e}=(0.0271,-0.6285,0.7774)
\end{aligned}
$$

For more details, the reader is referred to [10] and earlier references therein.

One particular feature of the DSM scheme is that all fermions lie on the same trajectory of the rotating vector $\left(x^{\prime}, y^{\prime}, z^{\prime}\right)$ so that at any scale the mass matrix for up and down fermion types have the same eigenvectors. This 


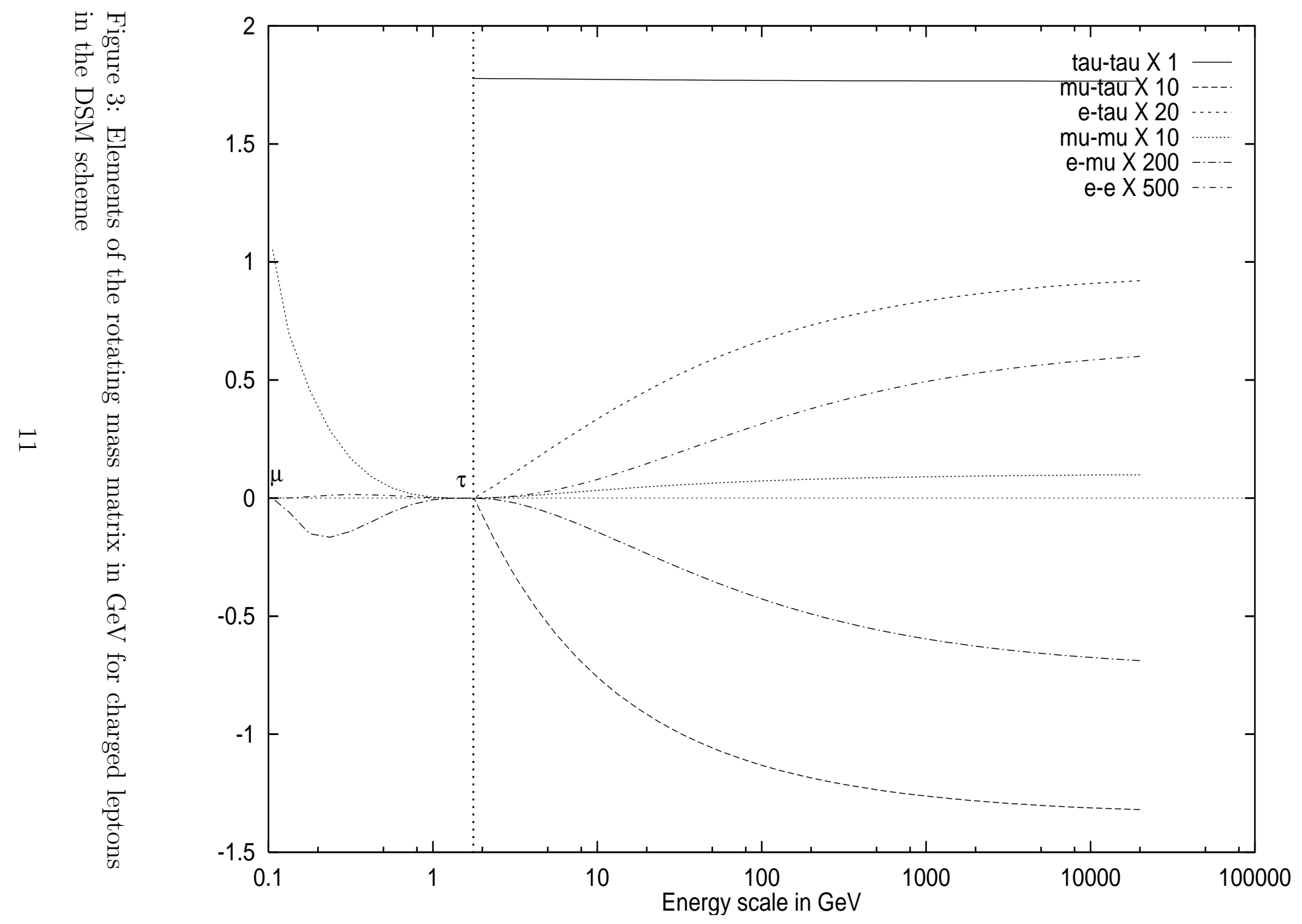


means that the $U$ and $D$ matrices in (1.1) and (1.2) can be simultaneously diagonalized, causing thus no de-diagonalization to each other via the electroweak Higgs loop effect. We recall that it was the latter which was what drove the rotation in the NSM case. Here instead all the rotation comes from the Higgs bosons associated with the broken 'horizontal' symmetry.

Comparing the actual values for the rotating mass matrices of the two schemes NSM and DSM, one finds the off-diagonal elements to be generally larger for the latter than the former, as we expected. Further, as can be seen in Figure 3, the mass matrix in the DSM scheme has quite an intricate structure with, for example, the off-diagonal elements passing through zero whenever the energy scale equals the mass of a physical fermion state, meaning that the mass matrix is there diagonal. This is a consequence of the SSD prescription by construction, and would apply also to the NSM had one chosen there also the SSD instead of the FSD prescription.

\section{Examples of Transmutational Decays}

Once given the rotating mass matrix as a function of the energy scale, it would appear that one has in principle already enough information to evaluate any implied transmutational effect. However, because of the mass matrix rotation leading to, among other things, some rather unfamiliar kinematics, adaptations to usual calculational procedures are required, which are not, at first encounter, entirely straight-forward. We find that this is true even in evaluating, for example, the simple Feynman diagrams of Figure 2 for the photo-transmutation of leptons that we have calculated. Therefore, in order to avoid clouding immediately the essentially simple general picture of transmutation with technical details, we relegate detailed calculations to other papers, e.g. on photo-transmutation to a companion paper [14], and limit ourselves here to qualitative estimates of widths for some illustrative examples in transmutational decays, which being single particle effects are simpler to analyse theoretically. Also, to avoid the complications of quark confinement, we shall deal here with only lepton transmutations, which are already of considerable physical interest, being constrained by some very stringent bounds in experiment.

(I) As a first example, consider the decays:

$$
Z^{0} \longrightarrow l_{\alpha} \bar{l}_{\beta}
$$

with $l_{\alpha} \neq l_{\beta}$ being different lepton states. As indicated already in Section 3, 
the amplitudes of such off-diagonal transmutational processes are expected to be suppressed with respect to those of their diagonal counterparts $Z^{0} \longrightarrow l_{\alpha} \bar{l}_{\alpha}$ by a factor of the form $\langle\alpha|m| \beta\rangle / E$, with $E$ being a measure of the typical energy carried by the fermion line, which we can take here to be of the order of (half) the $Z^{0}$ mass $M_{Z}$. The numerator $\langle\alpha|m| \beta\rangle$ represents the offdiagonal mass matrix element linking the lepton states $l_{\alpha}$ and $l_{\beta}$ at the scale of the reaction, which is again $M_{Z}$ in the present case. Given any choice for $l_{\alpha}$ and $l_{\beta},\langle\alpha|m| \beta\rangle$ can be read off for the DSM in Figure 3. Thus, for example, for the decay $Z^{0} \longrightarrow \tau^{-} \mu^{+}$the relevant matrix element $\langle\mu|m| \tau\rangle$ at the scale $M_{Z}=91 \mathrm{GeV}$ is seen to have value about $-0.11 \mathrm{GeV}$. For the NSM, the element $\langle\mu|m| \tau\rangle$ was said in Section 3 to vary by about 0.5 percent per decade change in energy. According to the FSD prescription adopted there, we have to first fix a scale at which the mass matrix is diagonal. If this is taken, for lack of any obvious better choice, to be at the $\tau$ mass, we obtain for $\langle\mu|m| \tau\rangle$ at $M_{Z}$ a value of about $0.01 \mathrm{GeV}$. To estimate the width for the decay $Z^{0} \longrightarrow \tau^{-} \mu^{+}$, the easiest way would be to compare it with either of the diagonal decays $Z^{0} \longrightarrow \tau^{-} \tau^{+}$or $Z^{0} \longrightarrow \mu^{-} \mu^{+}$. Given that the masses of the final state leptons are so much smaller than the decaying $Z^{0}$ mass, one can neglect the small differences in kinematics between the different decay modes and write simply:

$$
\frac{\Gamma\left(Z^{0} \longrightarrow \tau^{-} \mu^{+}\right)}{\Gamma\left(Z^{0} \longrightarrow \tau^{-} \tau^{+}\right)} \sim \frac{|\langle\mu|m| \tau\rangle|^{2}}{M_{Z}^{2}} .
$$

Putting in the above estimates of the matrix elements and the empirical value 3.36 percent [16] for the branching ratio of the diagonal $\tau^{-} \tau^{+}$mode, one obtains then for the branching ratio of the transmutational mode $\tau^{-} \mu^{+}$ an estimate of about $4 \times 10^{-8}$ for DSM and $4 \times 10^{-10}$ for NSM. Both of these estimates are much below the present empirical bound of $1.2 \times 10^{-5}$ listed in [16]. Similarly, estimates for the branching ratios of the other modes are obtained, giving for the $\tau^{-} e^{+}$mode a branching ratio of $4 \times 19^{-9}$ and for the $\mu^{-} e^{+}$mode $1.6 \times 10^{-11}$ for DSM. Again these are way below the present empirical limits listed in [16] of respectively $9.8 \times 10^{-6}$ and $1.7 \times 10^{-6}$. The estimates in the NSM for these last two modes are minuscule due to the very small values for the mass matrix elements linking $e$ to $\mu$ and $e$ to $\tau$, as will also be true for the following examples. They shall therefore henceforth be ignored except, for a particular reason to be explained, for (IV) below. As one can easily see, the reason that the transmutation rates are all so small in $Z^{0}$ decay is that the off-diagonal effects are much suppressed by the high energies involved which is here of the order of the $Z^{0}$ mass. 
(II) As a second example then, let us go to the other extreme in energy and consider the decay:

$$
\pi^{0} \longrightarrow \mu^{-} e^{+}
$$

which in $\pi^{0}$-decay is the only kinematically accessible transmutational leptonic mode. The same arguments as before suggests that we compare this with the diagonal mode $\pi^{0} \longrightarrow e^{-} e^{+}$giving a suppression in the amplitude of the former with respect to the latter by a factor of the form $\langle e|m| \mu\rangle / m_{\pi}$ where the off-diagonal mass matrix element is to be evaulated at the scale $m_{\pi}$. Here, the spins, parities and masses of the particles involved being such, a correction for the difference in kinematics between the two decays is warranted, which is made most easily by comparison with the analogous charged $\pi$ decays, giving thus:

$$
\frac{\Gamma\left(\pi^{0} \longrightarrow \mu^{-} e^{+}\right)}{\Gamma\left(\pi^{0} \longrightarrow e^{-} e^{+}\right)} \sim \frac{|\langle e|m| \mu\rangle|^{2}}{m_{\pi}^{2}} \frac{\Gamma\left(\pi^{ \pm} \longrightarrow \mu^{ \pm} \nu_{\mu}\right)}{\Gamma\left(\pi^{ \pm} \longrightarrow e^{ \pm} \nu_{e}\right)} .
$$

The matrix element $\langle e|m| \mu\rangle$ at scale $m_{\pi^{0}}=135 \mathrm{MeV}$ obtained from the calculation giving Figure 3 is about $3 \times 10^{-4} \mathrm{GeV}$. Hence, putting in the empirical value of $7.5 \times 10^{-8}$ for the branching ratio of the diagonal mode $\pi^{0} \longrightarrow e^{-} e^{+}$and that of $1.23 \times 10^{-4}$ for the branching ratio of $\Gamma\left(\pi^{ \pm} \longrightarrow e^{ \pm} \nu_{e}\right)$ [16], we have an estimate for the branching ratio of the transmuational mode $\pi^{0} \longrightarrow \mu^{-} e^{+}$of about $2.9 \times 10^{-9}$. This is barely an order of magnitude below the experimental bound of $1.72 \times 10^{-8}$ [16]. Indeed, one is saved here from a violation of the experimental limit only by the exceptionally small value of the off-diagonal mass matrix element due to the proximity of the reaction scale $m_{\pi^{0}}$ to the muon mass where the mass matrix is constrained to be diagonal by the SSD prescription.

(III) As a third example, let us consider at an intermediate scale:

$$
\psi \longrightarrow l_{\alpha} \bar{l}_{\beta} .
$$

Following the same procedure as above, one obtains:

$$
\frac{\Gamma\left(\psi \longrightarrow \tau^{-} \mu^{+}\right)}{\Gamma\left(\psi \longrightarrow \mu^{-} \mu^{+}\right)} \sim \frac{|\langle\mu|m| \tau\rangle|^{2}}{m_{\psi}^{2}} .
$$

The matrix element $\langle\mu|m| \tau\rangle$ for DSM as read from Figure 3 at the scale $m_{\psi}$ is about $0.03 \mathrm{GeV}$. Combined with the empirical value of about 6 percent [16] for the branching ratio of $\psi \longrightarrow \mu^{-} \mu^{+}$, one obtains an estimate of about $6 \times 10^{-6}$ for the transmutational $\tau^{-} \mu^{+}$mode, which is larger than in either of 
the two earlier examples. The corresponding estimate for the NSM is about $1 \times 10^{-8}$. Although no empirical limit is given in [16] for this particular mode, the estimates seem close enough to the sensitivity of present experiment to make a search for it worthwhile. Similar arguments applied to the modes $\psi \longrightarrow \tau^{-} e^{+}$and $\psi \longrightarrow \mu^{-} e^{+}$yield the estimated branching ratios $1.6 \times 10^{-7}$ and $6 \times 10^{-11}$. For these modes again, no empirical limits are given in 16 but the estimates seem in any case beyond present experimental sensitivity. The same anlysis can also be applied to $\Upsilon$ decay giving for the branching ratios of the modes $\tau^{-} \mu^{+}, \tau^{-} e^{+}$, and $\mu^{-} e^{+}$respectively the following estimates: $1.6 \times 10^{-6}, 6 \times 10^{-8}$, and $7 \times 10^{-11}$. Again, no empirical bounds for these modes are given in [16], but some, especially the $\tau \mu$ mode, will be worth searching for in future in e.g. $B$-factories.

(IV) Lastly, consider transmutational fermion decay, of which the prime examples would be:

$$
\mu^{-} \longrightarrow e^{-} \gamma
$$

and

$$
\mu^{-} \longrightarrow e^{-} e^{+} e^{-}
$$

proceeding via the Feynman diagrams in Figure 4 . The bound as given in

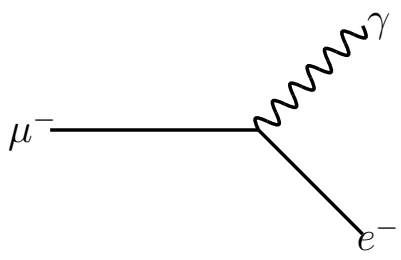

(a)

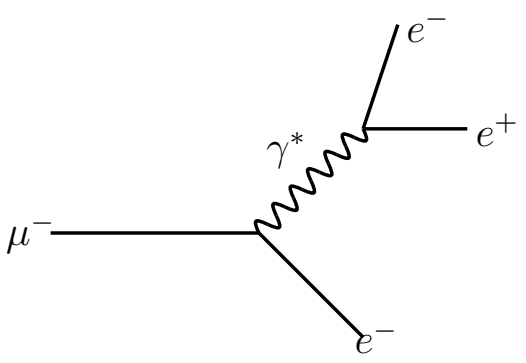

(b)

Figure 4: Transmutational $\mu^{-} \longrightarrow e^{-} \gamma$ and $\mu^{-} \longrightarrow e^{-} e^{+} e^{-}$decays

16] for the branching ratio of the decay (5.7) is an impressive $4.9 \times 10^{-11}$, and of the decay (5.8) is $1 \times 10^{-12}$. And these coming on top of a main decay mode of $\mu$, namely $\mu \longrightarrow e \nu \bar{\nu}$, proceeding by weak interactions with a width of only around $3 \times 10^{-10} \mathrm{eV}$, are a very stringent constraint indeed on any scheme which violates muon number. It is thus gratifying for us to note that transmutation in the DSM scheme survives this very dangerous-looking hurdle automatically. This comes about because the Feynman diagrams 4 
for the transmutational decays (5.7) and (5.8) are to be evaluated at the scale of the $\mu$ mass, and at that scale the mass matrix in the DSM scheme is diagonal. That it is so by virtue of the SSD prescription for defining lepton states was noted already in preceding sections and can be seen explicitly in Figure 3 by the vanishing of the off-diagonal mass matrix element $\langle e|m| \mu\rangle$ at the $\mu$ mass. Hence the DSM scheme actually predicts zero probability for this transmutational decay.

The same observation applies also to transmutational decays of $\tau$, for example:

$$
\begin{gathered}
\tau^{-} \longrightarrow e^{-} \gamma, \mu^{-} \gamma, \\
\tau^{-} \longrightarrow e^{-} e^{+} e^{-}, e^{-} \mu^{+} \mu^{-}, \mu^{-} e^{+} e^{-}, \mu^{-} \mu^{+} \mu^{-},
\end{gathered}
$$

since for these decays the analogous Feynman diagrams are to be evaluated at the scale of the $\tau$ mass, and at that scale the mass matrix in the DSM scheme is again diagonal giving thus zero transitions. This is also fortunate, for although not quite as stringent as those on (5.7) and (5.8), the bounds on the branching ratios of $(5.9)$ and (5.10) as given in [16] are still only of order $10^{-6}$ for a total $\tau$ width of around $3 \times 10^{-3} \mathrm{eV}$, which would be far from trivial to satisfy otherwise.

To illustrate how sensitive a test these lepton decays are to transmutation models, let us examine them in the NSM scenario. Here, as noted before, the rotation of the mass matrix is slower than in the DSM case giving thus usually smaller transmutation rates, especially for transmutations between $\mu$ and $e$. However, the mass matrix is required by the FSD prescription adopted to be diagonal only at some fixed mass scale. Suppose we choose this scale as the $\tau$ mass so as to make the transmutation rates vanish for both the decays (5.9) and $(5.10)$ and so satisfy the experimental bounds on them automatically. As the energy lowers to the scale of the $\mu$ mass, however, the mass matrix will have rotated and acquired nonzero off-diagonal elements. Indeed, according to (4.2) the element $\langle e|m| \mu\rangle$ would have acquired on running from the $\tau$ mass a value of around $1.3 \times 10^{-8} \mathrm{GeV}$, giving thus possibly nonzero rates to the decays (5.7) and (5.8). To estimates these rates following the previous procedure, let us compare for example (5.8) to the known main decay mode of $\mu$, namely:

$$
\mu^{-} \longrightarrow e^{-} \bar{\nu}_{e} \nu_{\mu}
$$

as given to first order by the Feynman diagram of Figure 5. The mass of the electron being so much smaller than that of the muon, we can ignore the 


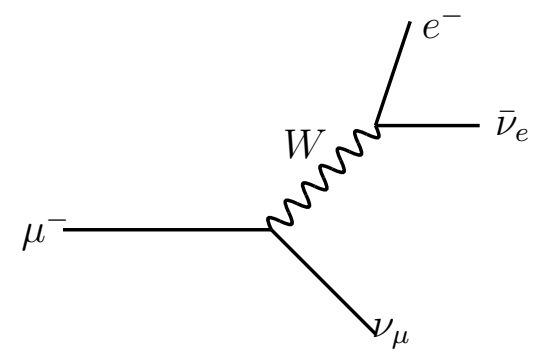

Figure 5: Feynman diagram for the decay $\mu^{-} \longrightarrow e^{-} \bar{\nu}_{e} \nu_{\mu}$

small difference in phase space between the two decays and write:

$$
\frac{\Gamma\left(\mu^{-} \longrightarrow e^{-} e^{+} e^{-}\right)}{\Gamma\left(\mu^{-} \longrightarrow e^{-} \bar{\nu}_{e} \nu_{\mu}\right)}=\frac{|\langle e|m| \mu\rangle|^{2}}{m_{\mu}^{2}} \frac{e^{2} / m_{\mu}^{2}}{g_{2}^{2} / M_{W}^{2}}
$$

where we have taken for a typical momentum for the decay to be of order of the muon mass. Inserting the above NSM value for $\langle e|m| \mu\rangle$ and the empirical values for the other quantities appearing in (5.12), one obtains a value of about $1 \times 10^{-3}$ for the ratio. This would be disastrous, being some 9 orders too large compared with the experimental bound given in [16 of order $1 \times 10^{-12}$. That this ratio turns out to be so large despite the very small NSM value for $\langle e|m| \mu\rangle$ is because of the large $W$ mass occuring in the diagram for the normal decay mode of $\mu$, or in other words, because we are in (5.12) comparing what is basically, in spite of being off-diagonal, still a photon-mediated process to a weak decay. The above blatant violation of the experimental bound is of course not the fault of the Standard Model but only of the naive fashion that it has been implemented in the NSM scheme since it could be easily avoided by, among other means, choosing a smaller value for the Dirac mass $m_{2}$ of the second heaviest neutrino which is still at present entirely unconstrained by experiment. Nevertheless, this example shows how sensitive these lepton decays are as a test of transmutation models, and how delicately the DSM scheme has managed to survive it.

Obviously, what really mattered for passing that test was what we called the SSD prescription for defining the lepton state vectors which stipulates that the mass matrix is to be diagonal at the mass of every lepton state. Had one chosen in the NSM scheme to define fermion states in this way instead of the FSD prescription adopted, it would have passed the test also. With the FSD prescription, however, it would not have mattered if one had 
chosen the scale for diagonalizing the mass matrix to be $m_{\mu}$ instead of $m_{\tau}$, for although one would then pass the test for the decay $\mu^{-} \longrightarrow e^{-} e^{+} e^{-}$, one would fail it for such decays as $\tau^{-} \longrightarrow \mu^{-} \mu^{+} \mu^{-}$. Indeed, running now from the $\mu$ mass, one would obtain a non-diagonal mass matrix at the $\tau$ mass with off-diagonal elements of the same order. The empirical bounds on transmutational $\tau$ decays are less stringent, e.g. the present bound on the branching ratio of $\tau^{-} \longrightarrow \mu^{-} \mu^{+} \mu^{-}$is only $1.9 \times 10^{-6}$ [16], but that would still mean a violation of the bound by some 6 orders in magnitude by the above estimate. One could of course impose in the NSM a further condition on the FSD prescription stating that the mass matrix should stop rotating altogether once below the chosen diagonalization scale, but one does not know of a good theoretical reason for doing so.

Firm conclusions cannot be drawn until detailed calculations for the various decay widths have been performed, which to us seem quite feasible in the near future. Tentatively, however, one can claim that the DSM predictions for transmutation survive present experimental bounds in all cases so far studied, while the NSM (with FSD) survives except perhaps for the noted $\mu$ or $\tau$ transmutational decays. In most cases, the predicted rates are some way below present experimental limits, but for the decays $\pi^{0} \longrightarrow e^{ \pm} \mu^{\mp}$, $\psi \longrightarrow \mu^{ \pm} \tau^{\mp}$ and $\Upsilon \longrightarrow \mu^{ \pm} \tau^{\mp}$ the estimated rates may soon be, if they are not already, within range of experimental sensitivity.

\section{Remarks}

The importance of possible fermion flavour violation has long been recognized and subjected to rigorous test by such experiments with ultra-high sensitivity as $\mu \longrightarrow e \gamma$ decay. The negative outcome to-date of all these tests conveys the impression that such violations can exist, if at all, only to a very small extent. However, that conclusion was reached in the days before it was realized that the fermion mass matrix actually rotates with the energy scale so that the fact that flavour is conserved at one scale, or that the mass matrix is there diagonal in the flavour states, does not necessarily mean that it will remain so at some other scales. That being the case, the accuracy, though impressive, of such experiments as $\mu \longrightarrow e \gamma$ searches, is not enough to rule out flavour violation in general but has to be supplemented by experiments at other energies. As our analysis above has shown, under some circumstances the mass matrix can acquire quite appreciable off-diagonal elements without being noticed in existing experiments. Hence, regardless of which scheme for 
mass matrix rotation one favours, it would be worthwhile for experimenters to perform routine checks under varying circumstances for the diagonality of fermion mass matrices by looking for effects of the type labelled in this paper as transmutations. With a rotating mass matrix, diagonality is no longer as sacrosanct as once believed.

As argued in the Introduction, that the mass matrix rotates forces on us a refinement of our definition of fermion flavour states. Given the sensitivity of such experiments as $\mu^{-} \longrightarrow e^{-} e^{+} e^{-}$searches, even the small rotations which may occur between the mass scales of two successive generations is enough to give huge violations of the experimental limits, as we have shown in the preceding section. It seems thus necessary either to define the fermion states in such a way as to ensure that the mass matrix is diagonal at the mass scales of all the fermion states, as was done in the SSD prescription, or else in a prescription like FSD to suppress altogether the rotations between fermion states, if not by some theoretical mechanism then by decree. For us, this conclusion has come as an agreeable surprise in that the SSD prescription which was originally invented just for internal consistency of our DSM scheme should turn out now to have the unexpected virtue of guaranteeing consistency with experiment on $\mu \longrightarrow e \gamma$ and $\mu^{-} \longrightarrow e^{-} e^{+} e^{-}$decays, which would otherwise be a difficult hurdle to survive. As we shall see in [14], the same property of the SSD prescription will save one also from certain unwanted pole structures in the amplitudes for the photo-transmutation of leptons.

The rotation of its mass matrix being weaker, the NSM scheme will give generally smaller transmutation effects than the DSM, i.e. provided that it has been amended with something like the SSD prescription for defining fermion states to avoid the noted probable inconsistencies. Indeed, in all the examples so far studied NSM transmutation effects seem to be beyond present experimental sensitivity. That being the case, if in the routine check on transmutation bounds an experiment comes up with an effect, then it is probably due to a rotation of the mass matrix driven by some forces of nature other than those currently considered in the Standard Model. This would be exciting in any case, besides possibly opening up a new window for probing into the generation mystery, as we have already said in the Introduction.

For us advocates of the Dualized Standard Model (DSM), the results of this paper and its companion [14 are something of a relief, at least temporarily. Since the whole DSM scheme relies heavily on the concept of a rotating mass matrix driven by dual colour forces, on which all its seemingly sensible predictions on the mass and mixing patterns of fermions also depend, it 
would be a little disastrous if the relatively fast mass matrix rotation required for the scheme's success predicts at the same time much larger transmutation effects than can be accommodated by present experiment. And, in contrast to flavour-changing neutral current (FCNC) effects [17, 18, 13] which also pose some hurdles for the scheme to overcome but depend on an unknown mass parameter, there is in transmutations no free parameter which could be adjusted as a loophole for escape. It is thus fortunate that a disaster does not seem so far to have happened although in some cases it has come rather dangerously close. Instead, one obtains as a bonus a whole new class of phenonmena, with quantitative predictions which, while remaining below present bounds, are still not that far off as to be entirely inaccessible to experiment. However, much more work will still be needed first, to make sure that disasters will not happen in circumstances not yet examined, and secondly, to identify specific cases where the scheme's predictions can be checked by experiment, if not immediately, at least in the not too distant future.

In general terms, what we have done in this paper is to raise some basic questions of concept forced on us by the rotation of the fermion mass matrix and attempt to answer some of them. Although in answering these questions we may have raised as many more, we hope at least to have clarified a little some of their implications.

\section{References}

[1] See e.g. B. Grzadkowski, M. Lindner and S. Theisen, Phys. Lett. B198, 64, (1987).

[2] H. Arason, D.J. Castaño, B. Kesthelyi, S. Mikaelian, E.J. Piard, P. Ramond and B.D. Wright, Phys. Rev. D46 (1992) 3945.

[3] N. Cabibbo, Phys. Rev. Lett. 10, 531 (1963); M. Kobayashi and T. Maskawa, Prog. Teor. Phys. 49, 652 (1973).

[4] T. Kafka, Nucl. Phys. B (Proc. Suppl.) 35, 427, (1994); M. Goodman, ibid 38, 337, (1995); W.W.M. Allison et al., Phys. Letters B391, 491, (1997).

[5] CHOOZ collaboration, M. Apollonio et al., Phys. Lett. B420, 397, (1997). 
[6] Y. Fukuda et al, Super-Kamiokande Collaboration, Phys. Lett. B433 (1998) 9; Phys. Lett. B436 (1998) 33; Phys. Rev. Lett. 81 (1998) 1562.

[7] Z. Maki, M. Nakagawa and S. Sakata, Prog. Theor. Phys. 28 (1962) 247.

[8] The idea of a possible "horizontal symmetry" linking generations is quite old. Examples of some early references are: F. Wilczek and A. Zee, Phys. Rev. Lett. 42, 421, (1979); A. Davidson and K.C. Wali, Phys. Rev. D20, 1195, (1979), D21, 787, (1980); T. Maehara and T. Tanagida, Prog. Theor. Phys. 61, 1434, (1979); T. Yanagida, Phys. Rev. D22, 1826, (1980).

[9] Chan Hong-Mo and Tsou Sheung Tsun, Phys. Rev. 57D, (1998) 2507, hep-th/9701120.

[10] José Bordes, Chan Hong-Mo and Tsou Sheung Tsun, hep-ph/9901440, Eur. Phys. J. C10 (1999) 63. A similar prescription, though in a different context, has been considered in [11].

[11] E. García, A. Hernández-Galeana, D. Jaramillo, W.A. Ponce and A. Zepeda, hep-ph/0006093.

[12] José Bordes, Chan Hong-Mo, Jacqueline Faridani, Jakov Pfaudler, and Tsou Sheung Tsun, Phys. Rev. D58, 013004, (1998), hep-ph/9712276.

[13] José Bordes, Chan Hong-Mo, Ricardo Gallego, and Tsou Sheung Tsun, hep-ph/9909321, Phys. Rev. D61, 077702, (2000).

[14] José Bordes, Chan Hong-Mo, Jacqueline Faridani, and Tsou Sheung Tsun, in preparation.

[15] V. Barger, S. Pakvasa, T.J. Weiler and K. Whisnant, Phys. Lett. B437 (1998) 107; A.J. Baltz, A.S. Goldhaber and M. Goldhaber, Phys. Rev. Lett. 81 (1998) 5730; R.N. Mohapatra and S. Nussinov, Phys. Lett. B441 (1998) 299; C. Jarlskog, M. Matsuda, S. Skadhauge and M. Tanimoto, Phys. Lett. B449 (1999) 240; Y. Nomura and T. Yanagida, Phys. Rev. D59 (1999) 017303; S.K. Kang and C.S. Kim, Phys. Rev. D59 (1999) 091302; H. Georgi and S.L. Glashow, Phys. Rev. D61 (2000) 097301.

[16] Particle Physics data booklet, (1998), from C. Caso et al, The Europ. Phys. J. C3 (1998) 1. See also the updates on the PDG's website (http://pdg.lbl.gov/). 
[17] José Bordes, Chan Hong-Mo, Jacqueline Faridani, Jakov Pfaudler, and Tsou Sheung Tsun, astro-ph/9707031, Astroparticle Phys. 8 (1998) 135.

[18] José Bordes, Chan Hong-Mo, Jacqueline Faridani, Jakov Pfaudler, and Tsou Sheung Tsun, hep-ph/9807277, Phys. Rev. D60 (1999) 013005. 